\title{
119. Aufbau und Abfolge von Gesprächsphasen
}

1. Begriffsklärung

2. Allgemeine Aspekte und Probleme der Phasierung

3. Forschungsansätze und Phasenmodelle

4. Die Phasen des Gesprächs

5. Forschungsprobleme und Desiderate

6. Literatur (in Auswahl)

\section{Begriffsklärung}

Der Begriff der Gesprächsphase wird in der Gesprächsforschung unterschiedlich verwendet. Es ist daher eine einleitende Begriffsklärung erforderlich. Als Phasen werden in der Literatur komplexe Einheiten innerhalb von Gesprächen bezeichnet, die wesentliche Handlungs- oder Themenkomplexe ausmachen, eine interaktionslogische Reihenfolge implizieren sollen und intern differenziert sind. Je nach Gesprächstyp gibt es spezifische Verlaufsformen von Gesprächen und damit spezifische Gesprächsphasen. Grobstrukturierungen des Gesprächs unterteilen das kommunikative Ereignis in die drei Phasen der Gesprächseröffnung, Gesprächsmitte und Gesprächsbeendigung (Henne/Rehbock 1995, 20), bzw. Eröffnungsphase, Kernphase und Beendigungsphase (Brinker/Sager 1996, 94) oder auch Anfangsphase, Gesprächsmitte und Beendigungsphase (Linke/Nussbaumer/ Portmann 1996, 282). Insbesondere die Gesprächsphase „Gesprächsmitte“ bzw. „Kernphase" zeichnet sich in der Regel durch eine hohe Komplexität aus, „d.h. eine vielfach geschichtete Gesprächshandlungsstruktur“"
(Henne/Rehbock 1995, 186). Das macht eine weitere Strukturierung der Gesprächsphase „Gesprächsmitte“ in Teilphasen nötig: „Teilphasen werden durch Themen- und Subthemenwechsel sowie durch situative Bedingungen ausgegliedert. Teilphasen bestehen aus aufeinander bezogenen Gesprächshandlungen (Henne/Rehbock 1995, 187). Anstelle des Begriffs der Phase (Henne/Rehbock 1995; Brinker/Sager 1996) wurden für Einheitenbezeichnungen auch andere Termini verwendet wie „Schema“ (Kallmeyer/Schütze 1976) oder „Handlungsmuster“ (Ehlich/Rehbein 1979), „script" (Müller 1984) etc. Entsprechend wurden auch für Binnensegmentierungen von Einheiten unterschiedliche Bezeichnungen gewählt: „Teilphasen, Gesprächsstücke, -teile“ (Henne/Rehbock 1995), „Abschnitte bzw. thematische Abschnitte“ (Schank 1981), ,Aktivitätskomplexe“ (Müller 1984), „Teilhandlungsschemata oder Handlungsschemakomponenten“ (Kallmeyer/Schütze 1976), „Pragmeme“ (Ehlich/Rehbein 1972, 1986), „Segmente" (Nofsinger 1991) u. a. m. Die Termini „Frame“ bzw. „Rahmen“ (Goffman 1977; Müller 1984) und „,script“ (Schank/Abelson 1977) bezeichnen kognitive Strukturierungen. Diese können sich zwar in Form von Erwartungen und Interpretationsrahmen der Gesprächsteilnehmer auf Interaktionen auswirken, sie sind aber als kognitive Größen keine Strukturierungseinheiten von interaktiven, konkreten Gesprächen. So kritisiert Nothdurft (1986a, 98) das script-Modell als zur „Rekonstruktion von Ideen von Handlungs- 
mustern geeignet, damit aber eben nicht zur Rekonstruktion der interaktiven Realität solcher Muster".

In der Forschung ist die Bezeichnung komplexer Einheiten von Gesprächen also uneinheitlich. Ein eher neutraler Begriff ist der der "Makrostruktur", der jedoch auch andere komplexe Phänomene erfassen kann als solche, die mit dem Ausdruck „Phase“ konnotiert werden (z. B. Interaktionsprofile (SpranzFogasy 1997), Stile (Hinnenkamp/Selting 1989) etc.; „Makrostruktur“ wird hier terminologisch nicht bezogen auf die spezifische Verwendung als semantische Tiefenstruktur i. S. van Dijks (1980a; b)). Der Ausdruck „Phase“ wiederum wird einmal strikter für linear organisierte Gesprächsmodellierung verwendet (s. Pothmann 1997, der verschiedene solcher Phasenmodelle anführt), zum anderen aber unspezifischer für Abschnitte unterschiedlicher Charakteristik und Größenordnung. Andere Konzeptualisierungen operieren terminologisch genauer mit den Begriffen Handlungsschemakomponenten (Kallmeyer/ Schütze 1976) und Handlungsmusterpositionen (Ehlich/Rehbein 1972).

Der Begriff „Phase“ kann verschiedene problematische Implikationen enthalten: $\mathrm{Er}$ suggeriert, dass Gesprächsphasen klar abgrenzbar und voneinander abgeschlossen sind, er suggeriert weiter, dass die Abfolge von Phasen linear ist und dass deren Vorhandensein für einen Interaktionstyp konstitutiv ist; darüber hinaus indiziert der Phasenbegriff eine klare Zuordnung von Gesprächsaktivitäten zu einer bestimmten Phase. Dagegen wurde in verschiedenen empirischen Arbeiten festgestellt (u. a. Pothmann 1997), dass eine exhaustive und eindeutige Zuordenbarkeit aller Aktivitäten zu bestimmten Phasen problematisch ist, dass die Annahme, alle Gesprächsbeteiligten befänden sich in derselben Phase, häufig nicht zutrifft, oder dass eine klare Abgrenzung von Phasen schwierig ist, zumal InteraktantInnen zeitweise zwei oder mehrere Phasen parallel bearbeiten können, was wiederum eine Zuordnung der Aktivitäten erschwert. Trotz der aufgeführten Probleme halten wir hier am Begriff der Gesprächsphase als allgemeinem Begriff fest. Er soll alle Modelle umfassen, die Makrostrukturen von Gesprächen erfassen, in denen schwerpunktmäßig zusammengehörende Aktivitäten durchgeführt werden, welche auf typischerweise vorangegangenen Aktivitäten aufbauen und die typischerweise nachfolgenden Aktivitäten zu Grunde liegen. Auf der allgemeinsten Ebene enthält der Begriff „Ge- sprächsphase" die für eine Bezugnahme wesentlichen Komponenten: Einheitlichkeit, Abgrenzbarkeit und Progredienz, die handlungs- oder themenbezogen konstituiert sind.

\section{Allgemeine Aspekte und Probleme der Phasierung}

Gespräche sind komplexe Einheiten sozialer Kommunikation mit einer Vielzahl interpretationsrelevanter Dimensionen und interner Strukturierungsgrößen. Interaktionsteilnehmer organisieren die formalen Strukturen eines Gesprächs wie etwa turns, Sequenzen, Korrekturen oder Reparaturen; sie reden über Sachverhalte, sie vollziehen Handlungen, indem sie fragen, bitten, beraten, schlichten etc. Neben einer qualitativen Gliederungsdimension, die sich an den Konstitutionsebenen eines Gesprächs - wie etwa der Gesprächsorganisation, der Sachverhaltsorganisation, der Identitäts- und Beziehungskonstitution, der Handlungsschemakonstitution etc. (zur Theorie der Interaktionskonstitution s. Kallmeyer 1985; Spranz-Fogasy 1997, 27 ff) - orientiert, gibt es noch eine quantitative Gliederungsdimension, auf der unterschiedlich große Einheiten in den Blick genommen werden: von Strukturierungen durch Gliederungssignale und Wörter (Mikrostrukturen), über Phrasen, turn constructional units, Beiträge (turns), Sequenzen (Mesostrukturen) bis hin zu Gesprächsphasen und ganzen Gesprächen (Makrostrukturen). Schank (1981) zufolge wird der Gesprächsverlauf durch Gliederungssignale und metakommunikative Formulierungshandlungen segmentiert (s. auch Techtmeier 1984, 109f); diese Aktivitäten sind nach Henne/ Rehbock (1995) aber bereits auf der (qualitativen) Ebene der Gesprächshandlungen und -schritte angesiedelt. Die Segmentierungsaktivitäten der SprecherInnen sind Ergebnis ihrer Einschätzungen der Interaktionssituation. Diese Einschätzungen können zwischen den Gesprächsbeteiligten während des Gesprächs und in extrakommunikativer Perspektive etwa nach Gesprächsabschluss - durchaus divergieren (Henne/Rehbock 1995, 265). Während seit Beginn der aufzeichnungsgestützten Untersuchung von Gesprächen viele Phänomene auf der Mikro- und Mesoebene untersucht wurden, blieb die Makroebene lange Zeit ein Desiderat empirischer Forschung (s. auch Sacks' Forderung nach der Analyse von „big packages“ (Sacks 1992), die Heritage 1985 wiederholt). Auch Levinson 
$(1990,307)$ fordert, ein Gespräch in seiner Gesamtorganisation zu betrachten, da es einige Gesprächssequenzen gibt, die die gesamten Austausche innerhalb einer spezifischen Gesprächsart strukturieren. Levinson selbst konzentriert sich auf Gesprächstypen, die eine solche Gesamtorganisation aufweisen, wie z. B. Telefongespräche, die klare Anfänge und sorgfältig strukturierte Beendigungen haben.

Erst in den achtziger Jahren sind dann auch Gespräche als Ganzes zum Gegenstand der Forschung geworden, die in ihrer jeweiligen kommunikativen Typik charakterisiert werden, oder auch komplexe Einheiten innerhalb von Gesprächen, die deren sozialen Handlungssinn und/oder thematische Organisation widerspiegeln (so z. B. für Beratungsgespräche, Schlichtungsgespräche, Verkaufsgespräche, Reklamationsgespräche usw.; im Überblick Becker-Mrotzek 1999). Für viele Gesprächstypen stehen genauere empirische Untersuchungen noch aus.

Bei Analysen der „Gesprächsmitte“ werden zur Phasierung v. a. zwei Dimensionen bzw. Interaktionsebenen in Anschlag gebracht: die Handlungs- und die Themenebene (Henne/ Rehbock 1995; Levinson 1990; Schank 1981; Techtmeier 1984). Der Aufgaben- bzw. der Themenwechsel soll danach zur Phasierung, zur Binnenstrukturierung genutzt werden, und die Analyse gesprächsorganisatorischer Eigenschaften wird als Mittel der formalen Bestimmung zur Unterstützung herangezogen. Gesprächsphasen werden aufgaben- bzw. handlungsbezogen rekonstruiert und/oder relativ zur thematischen Entwicklung als emergente Einheitenbildung erfasst. Es handelt sich dabei aber um eine analytische Trennung zweier Ebenen, die empirisch vielfältig verwoben sind (bspw. wenn in Schlichtungsgesprächen der Konflikt sich als Problem darstellt und die Handlungsorientierung sich deshalb von Schlichten zu Beraten ändert; s. Nothdurft/Spranz-Fogasy 1991). Während jedoch aufgabenbezogene Interaktionen wie bspw. in institutioneller Kommunikation eine übergreifende Systematik einzelner Aufgaben und deren Abfolge implizieren, kann dies für primär thematisch strukturierte Gespräche (wie bspw. small talk, freie Diskussion) nicht postuliert werden (s. u.).

\section{Forschungsansätze und Phasenmodelle}

In den unterschiedlichen Forschungsansätzen wurden divergierende Phasenmodelle entwi- ckelt: So arbeitet die Gesprächsanalyse sensu Kallmeyer und Schütze mit dem „SchemaKonzept" bzw. mit Handlungsschemata und Sachverhaltsschemata, die Funktionale Pragmatik bzw. die Diskursanalyse operiert mit dem Konzept des „Handlungsmusters“ (Ehlich/Rehbein 1979 u. a.) und schließlich gibt es auch Konzepte, die den Phasenbegriff selbst ansetzen (Henne/Rehbock 1995; Brinker/Sager 1996; Brons-Albert 1995; Hundsnurscher/Franke 1985) und sich auf Schank (1981) beziehen.

Im Folgenden wollen wir zunächst auf die Gesprächsphasenmodelle der Letztgenannten eingehen. Daran schließt sich die Darlegung des Handlungsmusterkonzepts von Ehlich und Rehbein an sowie des Handlungsschemamodells von Kallmeyer und Schütze. Bei all diesen Modellen handelt es sich stets um handlungsorientierte Gliederungsmodelle (3.1.). Auf Ansätze, die sich mit thematischer Phasierung befassen, gehen wir am Ende dieses Abschnitts ein (3.2.).

3.1. Nach Brons-Albert (1995, 87) stellen Phasenmodelle eine HANDLUNGSBEZOGENE Gesprächsgliederung dar, der zu entnehmen ist, aus welchen größeren Bestandteilen das Gespräch besteht und in welcher Reihenfolge diese auftreten. Die Phasengliederung soll Aussagen über die Struktur des Gesprächs geben. Marten $(1985,27)$ bezeichnet die Phasenmodelle als ,Skizze“ des chronologischen Verlaufs eines (Verkaufs-)Gesprächs.

Während Henne/Rehbock (1995) nur ganz allgemein den Begriff der Gesprächsphasen für Einheiten der Makrostrukturierung verwenden, orientieren Brinker/Sager (1996) ihre allgemeine analytische Beschreibung von Gesprächen entlang der Gesprächsphasen, wohingegen Hundsnurscher/Franke (1985) ein konkretes Gesprächsphasen-Modell für Verkaufsgespräche entwickeln.

Brinker/Sager (1996) unterscheiden zwischen der Struktur von Gesprächseröffnungen, -beendigungen und der Struktur von Kernphasen eines Gesprächs. Die Grundstruktur von Gesprächseröffnungen und -beendigungen und einige Realisierungsmöglichkeiten explizieren die Autoren anhand von Telefongesprächen, in denen bestimmte Paarsequenzen als Eröffnungsritual und Beendigungsritual konstitutiv sind. Hingegen gehen sie bei der Behandlung der Kernphase von Gesprächen nur auf begriffliche und methodische Grundlagen für die Analyse von Kernphasen ein. Sie betonen die enge Ver- 
bundenheit der thematischen Ebene und der Handlungsebene, die beide der Anwendung von Segmentierungsverfahren dienen, und behandeln auch Schwierigkeiten der Segmentierung der thematischen Ebene. Dabei orientieren sie sich an Schank (1981).

Schank (1981) beschreibt die ganzheitlichen Strukturen von Kurzberatungen mit Hilfe von „Handlungsplänen“: „Der Begriff des Plans kann hier definiert werden als eine antizipierte Abfolge von Teilzielen und die auf die Realisierung derselben gerichtete $\mathrm{Ab}$ folge von Intentionen, durch die ein gegenwärtig bestehender Zustand in einen kontrafaktischen erstrebten Zielzustand überführt werden soll. Pläne können selbst hierarchisch gestuft ineinander eingebettet sein. Sie können also im Verhältnis von Metaplänen und Unterplänen zueinander stehen" (Schank 1981, 181). Das Konzept des Handlungsplans ist sprecherorientiert und nicht interaktiv, Handlungspläne und Teilziele werden hypothetisch angenommen, also deduktiv gewonnen ,indem man sich klarzumachen sucht, was in den Kurzberatungen (...) kommunikativ abläuft" (ebd., 186). Ergebnis der Arbeit von Schank ist die Erstellung eines Handlungsplans „Beraten“, dessen Teilziele regelhaft erwartbar in Gesprächen auftreten, wobei sie diskontinuierlich in ausdrucksseitigen Teilen realisiert werden (ebd., 268).

Hundsnurscher/Franke (1985) haben für den Gesprächstyp Verkaufsgespräche ein konkretes Phasenmodell entwickelt. Über die Bestimmung der kommunikativen Ziele der Interaktanten wird eine theoretisch-deduktive Zerlegung des Verkaufsgesprächs in konstitutive Einheiten vorgenommen, so genannte „funktionale Phasen“ (ebd., 79), die eine funktional ausgerichtete Einteilung darstellen: (i) Eröffnungs- oder Kontaktphase, (ii) Argumentations- oder Überzeugungsphase, (iii) Aushandlungs- oder Preisfestlegungsphase sowie (iv) Abschlussphase (Franke 1985, 54). Franke spricht von Phaseneinteilungen, die sich an der Frage orientieren, welche Themen und Subthemen während eines Verkaufsgesprächs von den Interaktanten behandelt werden. Nach ihm handelt es sich also bei der Phasengliederung um den Versuch, die Stadien von Verkaufsgesprächen thematisch zu fixieren und voneinander abzugrenzen. Auffällig ist der Gedanke der Linearität der Gesprächsverläufe, die den Verkaufsgesprächen unterstellt wird. Hinzu kommt, dass die genannten Phasen (i-iv) als konstitutiv für das Verkaufsgespräch angenommen werden.
Pothmanns kritische empirische Überprüfung des Gesprächsphasen-Modells von Verkaufsgesprächen, das Hundsnurscher/Franke entwickelt haben, ergibt Folgendes (1997, $50 \mathrm{f})$ : Die Phasen in tatsächlichen Verkaufsgesprächen verlaufen nicht linear und nicht chronologisch, sondern vielfach zirkulär. Phasen können abgebrochen und wieder aufgenommen werden und die einzelnen Gesprächsbeteiligten können sich in unterschiedlichen Phasen befinden. Dadurch kommt es zu einem Grenzziehungsproblem zwischen Phasen und zu einem Zuordnungsproblem von Äußerungen bzw. Äußerungsteilen gegenüber den Phasen - manche Äußerungen können mehreren Phasen zugeordnet werden. Darüber hinaus sind nicht alle von Hundsnurscher/Franke (1985) genannten Phasen für das Verkaufsgespräch konstitutiv, insbesondere ii) Argumentations- oder Überzeugungsphase und (iii) Aushandlungs- oder Preisfestlegungsphase können ganz wegfallen, andere hinzukommen (z. B. die Wunschbekundung seitens des Kunden). Pothmann kommt zu dem Ergebnis, dass das Phasenmodell jedenfalls nicht die Spezifika seines Datenmaterials (Schuhverkaufsgespräche) wie z. B. Bedarfsbefragung und -definition sowie die Kaufrealisation - abbildet (1997, 40). Das Handlungsmusterkonzept wurde von Ehlich/Rehbein (1972) entlang der Beschreibung einer Essensbestellung in der gesellschaftlichen Institution „Speiserestaurant" entwickelt und in der Folge für weitere Situationen (Unterrichtsgespräche in der Schule, Verkaufsgespräche etc.) spezifiziert und differenziert (Ehlich/Rehbein 1972, 1979, 1986; Rehbein 1995 u. a.). Allerdings heißen die einzelnen Handlungen bzw. die konstituierenden Einheiten zunächst noch Pragmeme $(1972,224)$ und eine Abfolge von Handlungen wird Praxeogramm genannt. Der Begriff des „Handlungsmusters“ (Rehbein 1977; Ehlich/Rehbein 1979) bezieht sich auf kommunikative und damit soziale Tiefenstrukturen mit dem Anspruch, die gesellschaftlich ausgearbeitete allgemeine Form wiederzugeben (Rehbein 1977, 183). Zentrale Bestimmung des Musters ist der Zweck und damit die gesellschaftliche Funktion, die Orientierung erfolgt entlang der Funktionen der Handlungen, die mentaler, sprachlicher und aktionaler Art sein können. In den Arbeiten zur Institution „Schule“ haben Ehlich/Rehbein (1986) ihre Musterabläufe anhand empirischen Materials erarbeitet. Das Konzept des Handlungsmusters enthält ein breites Spek- 
trum von Handlungsmöglichkeiten, die sich an Entscheidungsknoten weiter verzweigen; instruktiv ist dieses Konzept vor allem für die Analyse komplexer Sprecherbeiträge und Sequenzen mittlerer Größe.

Das Konzept des Handlungsschemas geht auf Kallmeyer/Schütze (1976) zurück. Dem zugrunde liegt die Vorstellung, dass die Gesprächsanalyse Gespräche versteht ,als eine komplexe Hierarchie von Aufgaben, die von den Kommunikationspartnern gemeinsam zu bewältigen sind. Für jeden Gesprächstyp lässt sich - gestützt auf umfangreiche empirische Korpusanalysen - eine spezifische Aufgabenstruktur herausdestillieren. Diese hierarchische Aufgabenstruktur wird als Handlungsoder Interaktionsschema bezeichnet" (Fiehler/Kindt/Schnieders 1999, 134). „Einzelne Aufgaben des Schemas können in mehreren 'Anläufen' oder Runden an verschiedenen Stellen des Gesprächs bearbeitet werden“ (ebd.).

Kallmeyer hat 1985 ein Handlungsschema für Beratungsgespräche entwickelt, das die Aufgabenstruktur des Beratungsgesprächs erfasst, die für diesen Gesprächstyp konstitutiv ist. Dabei legen die GesprächsteilnehmerInnen die Reihenfolge der Aufgabenbearbeitung nach drei Prinzipien der Interaktionskonstitution fest (Kallmeyer 1985, 96f): (i) Prinzip der logischen Abfolge: Folgeschritte können erst dann erfolgreich vollzogen werden, wenn die vorangehenden Schritte die notwendigen Voraussetzungen erbracht haben; (ii) Prinzip der Zerlegung komplexer Aufgaben und Anpassung der Teilfokussierung an die jeweiligen lokal wirksamen Kontextbedingungen; (iii) Koordinierungsprinzip der Interaktion; mit diesem Prinzip sind Verfahren der Bedeutungskonstitution und des Handlungsvollzugs verbunden, die darauf hinauslaufen, dass manifeste Definitionen von Aktivitäten erst im Vollzug dieser Aktivität allmählich herausgearbeitet und häufig auch erst nachgeliefert werden.

Diese Prinzipien der Interaktionskonstitution ermöglichen ein flexibles Umgehen mit den Handlungsschemakomponenten, die zwar hierarchisch strukturiert, aber nicht chronologisch linear festgelegt sind, sondern sich an der Interaktionslogik des konkreten Gesprächs orientieren. Das Grenzziehungsproblem zwischen den einzelnen Phasen besteht nicht mehr, da es im Kontext des Handlungsschemakonzepts möglich ist, gerade die Übergänge explizit zu untersuchen. Auch die Zuordnung der Gesprächsbeiträge zu einzel- nen Phasen entfällt, da Äußerungen als multifunktional betrachtet werden, d. h. mehrere Aufgaben können gleichzeitig bearbeitet werden.

Pothmann (1997, 101), der das Konzept des Handlungsschemas mit dem Phasenmodell von Hundsnurscher/Franke (1985) vergleicht, kommt $\mathrm{zu}$ folgendem Ergebnis: „Demnach ist das Phasenmodell als Spezialfall des Handlungsschemas einzuordnen, da das Handlungsschema (...) auch das beschreiben kann, was das Phasenmodell nicht mehr gewährleistet."

Einige weitere Phasierungskonzepte eignen sich weniger für die makroskopische Strukturierung ganzer Gesprächsverläufe. Stellvertretend soll hier auf Nofsinger (1991) eingegangen werden. Er entwickelt eine Phasengliederung von Argumentationen und Erzählungen anhand von adjacency pairs (Nachbarschaftspaare) mit möglichen Expansionen und demonstriert sie an kurzen Transkriptausschnitten, wobei er anmerkt, dass damit auch durchaus längere Ausschnitte gegliedert werden können. Er berücksichtigt so zwar die Interaktivität von Gesprächsausschnitten, lässt aber übergreifende Orientierungen und größere prozessuale Entwicklungen außer Acht. Zusammenfassend lässt sich sagen, dass die hier dargestellten Konzepte handlungsbezogener Einheitenstrukturierungen in unterschiedlichem Maß den Anforderungen an ein Phasierungsmodell nach interaktionslogischer Linearität bzw. der Entfaltbarkeit mit einer flexiblen Dynamik, nach Hierarchisierbarkeit und gleichzeitiger Revidierbarkeit dieser Hierarchie oder nach interaktionstyp- und situationsspezifischer Variierbarkeit nachkommen. Andere Konzepte wiederum beschreiben nur Gesprächsausschnitte oder einzelne Ebenen, und die Komplexität von Interaktion kann daher nicht adäquat abgebildet werden. Viele Phasenmodelle, die deduktiv-theoretisch entwickelt wurden, behaupten hingegen die konstitutive Bedeutung der dargestellten Phasen, ihre homogene Linearität und exhaustive Erfassung sowie die Exklusivität und Kohärenz der Phasen, und sie beanspruchen Spezifizität für die jeweilige Interaktionstypik - diese Ansprüche können jedoch einer empirischen Überprüfung vielfach nicht standhalten. Hingegen bewähren sich die beiden empirisch angelegten Phasierungsmodelle, das Handlungsmuster-Konzept und das HandlungsschemaModell, in Bezug auf die Charakterisierung von komplexen Einheiten in ihrer Gesamtheit, in Bezug auf die Erfassung der Komplexität 
und der Spezifik von Gesprächstypen, in Bezug auf die Beschreibung der inneren Strukturierung und des Ablaufs von verbalen Interaktionen und Analyse von Kommunikationsproblemen.

3.2. Für die primär THEMENORIENTIERT strukturierten Phasenmodelle gilt Vergleichbares wie für die handlungsorientierten Modelle. Für die Analyse allgemeiner thematischer Organisation (topic organization) von Gesprächen hat Sacks (1971) zwei Formen der Themenprogression unterschieden: den Übergang eines Themas in ein anderes (,stepwise topical movement") und die Initiierung eines neuen Themas nach Abschluss eines vorangehenden Themas (,,boundaried topical movement"). Diese Formen der thematischen Progression können im Rahmen von handlungsschemagebundenen Aktivitäten entfaltet werden (bspw. bei der Präsentation eines Problems in Beratungsgesprächen; s. Nothdurft 1984), sie können aber auch gegenüber einer schwach ausgebildeten Aufgabenstruktur, wie z. B. bei Unterhaltungen, die Phasierung eines Gesprächs dominieren.

Zur analytischen Rekonstruktion der thematischen Phasierung wurde eine Reihe von Vorschlägen entwickelt. Schank (1981) bestimmt „thematische Abschnitte" als das, was zwischen zwei Themenwechseln liegt, was von den GesprächsteilnehmerInnen mit Gliederungssignalen und Formulierungshandlungen markiert wird. Er knüpft dabei an den Fokusbegriff Goffmans (1971) an: „Thema ist das intentionale Objekt im Fokus einer zentrierten Interaktion, soweit dieses explizit verbalisiert wird" (1981, 22). Brinker (1988, $27 \mathrm{f})$ kritisiert die Konzeption thematischer Abschnitte als zu unpräzise und schlägt eine semantisch-strukturelle Analyse vor, die auch implizite bzw. präsupponierte Themen systematisch erfasst: Das Thema ist der Kern des Gesprächsinhalts, der Grund- oder Leitgedanke eines Gesprächs oder einer Gesprächsphase. Im Gespräch werden dann meist mehrere Themen realisiert, es gibt eine Themenhierarchie, die durch ein Ableitbarkeitsprinzip und ein Kompatibilitätsprinzip ermittelt werden kann ( $\rightarrow$ Art. 18 und 120). Dittmar (1988, 71) schlägt eine Gliederung des Gesprächs in thematische Felder vor, dem je ein Schlüsselwort zugeordnet werden kann. Ein thematisches Feld wird in thematischen Etappen interaktiv bearbeitet, wobei thematische Wanderungen, die nicht als linear anzunehmen sind, auftreten.
Die genannten Ansätze sind jedoch hinsichtlich ihres Themenbegriffs und v. a. hinsichtlich einer prozessualen Rekonstruktion der thematischen Entwicklung unterbestimmt. Wolf (1991) diskutiert kritisch die verschiedenen Ansätze zum Thema-Begriff und seinen Derivaten wie „Inhaltskern“, „Redegegenstand“ bzw. „Gegenstand der Kommunikation" und verweist auf die Schwierigkeiten, ein Thema im konkreten Gespräch festzumachen. Sie kommt zu folgendem interaktiven und prozessualen Thema-Konzept: „Wir unterscheiden zwischen Thema und Kommunikationsgegenstand als zwei eng aufeinander bezogenen Kategorien der Textkonstituierung. Thema wird verstanden als der spezifische Aspekt, unter dem die interaktive Verarbeitung des (kognitiv repräsentierten, Anm. d. Verf.) Kommunikationsgegenstandes erfolgt und den die Partner während des Gesprächs permanent aushandeln" (ebd., 62).

Ähnlich sieht es Levinson (1990, 312 f), der die traditionellen Ansätze (z. B. den Referenz-Ansatz von Putnam 1958 oder den Konzept-Ansatz von Beaugrande/Dressler 1981) kritisiert. Levinson plädiert statt dessen für eine Betrachtung der Gesamtorganisation des Gesprächs und stellt fest: „Es ist einfach so, dass man nicht davon ausgehen kann, dass thematische Kohärenz in einem unabhängig berechenbaren Vorgehen besteht, das (beispielsweise) dafür sorgt, dass aufeinanderfolgende Äußerungen gemeinsame Referenten haben. Vielmehr ist thematische Kohärenz etwas, was über Redebeiträge hinweg durch die Zusammenarbeit der Gesprächsteilnehmer hergestellt wird“ (Levinson 1990, 313).

Thematische Entwicklungen werden auch aus der Perspektive musterhafter Darstellungsformen betrachtet. Als Standardvariante einer solchen Phasierung gelten Erzählsituationen bzw. Erzählungen. Labov/Waletzky (1973) haben dafür ein lineares Abfolgemodell von Erzählungen entwickelt mit den Komponenten Orientierung, Komplikation, Evaluation, Auflösung, Coda, wobei diese Strukturierung zurückgeht ,auf das Prinzip der temporalen Folge, d. h. dem direkten Abbildverhältnis zwischen Ereignisabfolge und Äußerungsabfolge" (nach Quasthoff 1980, 32). Quasthoff problematisiert u.a. die lineare Abfolge der Strukturelemente und stellt fest, dass diese sich u. a. in empirischer wie theoretischer Hinsicht als problematisch erwiesen hat (ebd.). Normalformen von Erzählungen müssen nicht alle von Labov und Waletzky entwickelten Komponenten beinhal- 
ten, auch hier gilt, dass einige Komponenten, wie beispielsweise die Orientierung, über die gesamte Erzählung hinweg realisiert werden, oder aber je nach Kenntnisstand oder Vertrautheitsgrad der Gesprächsbeteiligten ganz wegfallen können (s. hierzu Quasthoff 1980, 90).

Andere Analysen der Darstellungsstruktur von Erzählungen und Argumentationen zielen auf die Rekonstruktion von Schemata der Sachverhaltsdarstellung (Kallmeyer/Schütze 1977; Schütze 1978). Sie orientieren sich jedoch gerade nicht an prozessualen Phasierungseigenschaften, sondern stellen Zugzwänge und kognitive Strukturen in den Vordergrund.

Festzuhalten bleibt, dass in den verschiedenen Ansätzen eine Strukturierung entlang thematischer Entwicklungen zwar unter Bezug auf die Gesprächsempirie konstatiert wird, Modelle einer thematisch organisierten Phasierung jedoch bislang nicht vorhanden sind.

\section{Die Phasen des Gesprächs}

Die Darstellung von Forschungsansätzen und Phasenmodellen soll im Folgenden ergänzt werden durch exemplarische Charakterisierungen von Gesprächsphasen. Aufgrund der eben dargestellten Forschungslage beschränken wir uns auf handlungsbezogene Analysen der Gesprächsphasen. Wir orientieren uns dabei, wie in der Forschung üblich, am Dreischritt Gesprächseröffnung, Gesprächsmitte und Gesprächsbeendigung (s. 2.), wobei wir die Gesprächseröffnung und -beendigung, nicht zuletzt ihrer einfachen Strukturierung und ihrer Komplementarität wegen, zuerst und zusammenhängend behandeln.

4.1. ERÖFFNUNG UND BEENDIGUNG: Die Zugangsprobleme der komplexen Einheit „Gespräch" spiegeln sich in der Vielzahl von Arbeiten, die sich mit Gesprächseröffnung und -beendigung befassen (Schegloff 1968; Schegloff/Sacks 1973; Jäger 1976; Berens 1981; Werlen 1984 etc.). Phasen der Gesprächseröffnung und -beendigung sind leicht erkennbar, weil sie mit operationalisierbaren (Laut-)Phänomenen einhergehen, „sie leisten die Ausgrenzung der Interaktionseinheit 'Gespräch' aus dem 'Fluß des Verhaltens'" (Henne/Rehbock 1995, 262) und sie sind klar strukturierte Einheiten. Vielfach wurden sie anhand von Telefongesprächen untersucht, also unter Beschränkung auf den akustischen Kanal.

In der Phase der Gesprächseröffnung leisten die GesprächsteilnehmerInnen verbal, z.B. durch Grußformeln, und nonverbal, z. B. durch Blickkontakt, die wechselseitige Identifizierung und stellen gemeinsam Gesprächsbereitschaft her; beides ist Bestandteil der Situationsdefinition, die die Beteiligten gemeinsam aushandeln (zum Situationskonzept der Gesprächslinguistik s. $\rightarrow$ Art. 111; zur Relevanz der wechselseitigen Wahrnehmung als dem für die Interaktionseröffnung zentralen Aspekt s. Hausendorf 1992). Bei den in diesem Zusammenhang vielfach untersuchten Telefongesprächen bestehen die Eröffnungen häufig aus Nachbarschaftspaaren, in denen es um die wechselseitige Identifizierung und Begrüßung der GesprächsteilnehmerInnen geht - die bei Vertrautheit der Stimmen auch zusammenfallen kann - sowie um die Anrufsbegründung, die gleichzeitig die Überleitung zur thematischen Organisation des Gesprächs darstellt. Die weitere gemeinsame Aushandlung von Handlungsaufgaben und Gesprächsthemen markiert dann den Übergang zur Kernphase bzw. zur Mitte des Gesprächs. Bei face-to-face-Gesprächen hingegen finden wechselseitige Identifizierungen nur im Bedarfsfall bei einander unbekannten Personen statt. Die Dauer der Eröffnungsphase kann stark variieren; sie ist von verschiedenen Faktoren abhängig: dem Bekanntheitsgrad der Teilnehmenden und deren Zeitbudget, der Öffentlichkeit oder Privatheit der Gesprächssituation, dem Zeitpunkt des letzten Kontakts etc. In beruflichen Gesprächen wird ein relativ rasches Zur-Sache-Kommen erwartet, wohingegen bei einem privaten Kontakt zweier Vertrauter, die sich lange nicht mehr gesehen haben, eine relativ lange Eröffnungsphase erwartbar ist, die schleichend in die Phase der thematischen Organisation übergeht, in der Befindlichkeiten nachgefragt werden, wechselseitig Informationen aktualisiert werden etc. In wieder anderen Gesprächssituationen, so z. B. bei Vertrauten, die in beruflichen Situationen zusammenkommen, kann die Eröffnungsphase der Vergewisserung der Vertrautheit dienen, und der Übergang in die Kernphase gleichzeitig einen neuen, z. B. geschäftlichen Rahmen etablieren, bspw. wenn ein Freund auch als Arzt in Anspruch genommen 
wird. Gerade Rahmenwechsel von einer informellen zu einer formellen, häufig institutionellen Gesprächssituation können gar durch eine zweite Eröffnung, einer offiziellen Begrüßung etwa, markiert werden, die auch gleichzeitig die neue Situation - eine Gerichtsverhandlung, eine Parlamentssitzung herstellt. Am Ende der 'formellen Phase' wird diese auch durch entsprechende Beendigung wieder außer Kraft gesetzt, und es schließt sich häufig eine informelle Phase mit einer zweiten Beendigung an. Spätestens hier stellt sich die Frage nach der Segmentierung der Einheit 'Gespräch' in die drei Phasen der Gesprächseröffnung, Gesprächsmitte und -beendigung.

Komplementär zur Gesprächseröffnung wird die Phase der Gesprächsbeendigung durch Handlungs- und Themenbeendigungsinitiativen eingeleitet und häufig durch entsprechendes nonverbales Verhalten, z. B. durch Aufstehen, Zusammenpacken etc. begleitet. Gesprächsbeendigungen sind insofern heikel, als ,überhastige wie überlangsame Bedingungen unwillkommene Inferenzen für die gesellschaftliche Beziehung zwischen den Gesprächsteilnehmern bergen können" (Levinson 1990, 315). Daher benötigen Beendigungen in der Regel sorgfältige Vorbereitungen wie beendigungsimplizierende Themen und entsprechende Ratifizierungen, mehrere wechselseitige Übergangs- und Vergewisserungsbeiträge, die die Möglichkeit zulassen, zugunsten einer neuerlichen Expansion der Kernphase oder einer gänzlich neuen thematischen oder handlungsbezogenen Orientierung die Beendigungsphase bis auf Weiteres zurückzustellen, und einen abschließenden Austausch von Schlusselementen. Die Gespräche selbst werden dann i.d. R. durch Verabschiedung qua paarweise auftretender Grußformeln beendet. Schegloff/Sacks (1973) unterscheiden verschiedene Arten von Beendigungsinitiativen: Resümee des Gesprächs, Terminabsprachen, Dank, Austausch von guten Wünschen, Grußaufträge, Abschiedsfloskeln etc. Linke/Nussbaumer/Portmann (1996) ergänzen diese Liste um 'Ausblicke auf zukünftige Handlungen der Gesprächspartner' und 'Wertungen der Gesprächsereignisse', wobei bis auf die Grußformeln die meisten Handlungen fakultativ sind. Levinson (1990) weist darauf hin, dass bei der Gesprächsbeendigung gerne Aspekte der Gesprächseröffnung (z. B. thematische Wiederaufnahme der persönlichen Befindlichkeit,
Entschuldigung für die Störung) oder des Gesprächsverlaufs insgesamt aufgegriffen werden, wie es bei der Wertung der Gesprächsereignisse der Fall ist. Für viele neu zu konstituierende Gesprächssituationen (z. B. Fahrkartenverkauf) oder bei dauerhafter räumlicher Nähe (z.B. längere gemeinsame Autofahrten) finden aber nur minimale oder gar keine verbalen Eröffnungs- und Beendigungsaktivitäten statt.

4.2. Die GESPRÄCHSMITTE erweist sich im Vergleich zu den relativ einfach strukturierten Eröffnungen und Beendigungen als wesentlich komplexer organisiert. Die Aufgabenstellungen erweitern sich für die Gesprächsteilnehmer um ein Vielfaches bezogen auf alle Ebenen der Interaktionskonstitution (Konstitution eines Handlungsschemas, damit verknüpfte Darstellungsaufgaben, Differenzierung der Beteiligungsrollen etc.).

Die Phasierung eines Gesprächs auf der Grundlage des Handlungsschema-Modells sensu Kallmeyer und Schütze (s. 2. und 3.1.) soll in diesem Abschnitt anhand eines Beispiels präsentiert werden. Die Herausarbeitung des Handlungsschemas ist ein empirischer Prozess; mit der Zahl der Analysen wird die Modifikation des Handlungsschemas zunehmend geringer, wobei es jeweils neuen, spezifischen Analysematerialien entsprechend adaptiert werden kann (s. Kallmeyer 1985; auch Deppermann 1999; Pothmann 1997).

Fiehler/Kindt/Schnieders (1999, $134 \mathrm{ff})$ haben ein Handlungsschema für Reklamationsgespräche entwickelt, das hierarchisch gegliedert ist, das von allgemeinen zu konkreten Aufgaben wandert und die Spezifik des Gesprächstyps „Reklamationsgespräch“ abbildet. Es wird hier seiner Kürze und Prägnanz wegen als Beispiel dargestellt, auf weitere und ausführlichere Beispiele von Handlungsschemaanalysen verweisen wir am Ende dieses Abschnitts. Am Anfang der Interaktion stehen Aufgaben wie Begrüßung, Identifizierung und vorgreifende Verdeutlichung des Aktivitätstyps. Auf der allgemeinsten Ebene lassen sich Aufgaben der „Emotionsund Beziehungsbearbeitung“ und der „Bearbeitung des sachlichen Problems" (1. Hierarchiestufe) unterscheiden, die durchaus miteinander verwoben sein können. Zur Aufgabe der Emotionsbearbeitung (2. Hierarchiestufe) zählen die „Manifestation von Emotionalität“" (3. Hierarchiestufe) - z. B. in Vorwür- 
fen, Aufgebrachtheit bei der Problemdarstellung etc. (4. Hierarchiestufe) - wie auch die „Reaktion auf die Emotion“ des Kunden (3. Hierarchiestufe), z. B. durch die Kundgabe von Anteilnahme und die Bearbeitung der Vorwürfe (4. Hierarchiestufe). Neben der „Emotionsarbeit" steht die „Beziehungsarbeit" (2. Hierarchiestufe) z. B. durch Imagepflege.

Auf der ersten Hierarchiestufe findet sich neben der Emotionsbearbeitung die „Bearbeitung des sachlichen Problems" mit den Komplexen „Problemexplikation und Voraussetzungsklärung" sowie „Problemlösung" (2. Hierarchiestufe). Auch die Aufgaben der zweiten Hierarchiestufe werden weiter in Teilaufgaben konkretisiert, auf die wir nicht weiter eingehen wollen; die Struktur des Handlungsschemas mit den verschiedenen Hierarchie-Ebenen dürfte mit der bisherigen Darlegung ausreichend deutlich geworden sein.

Nicht alle Aufgaben müssen aber in jedem Reklamationsgespräch bearbeitet werden. Die Aufgabenbearbeitung findet auch nicht zwangsläufig in einer bestimmten Reihenfolge statt, sondern kann während des gesamten Reklamationsgesprächs wiederholt aufgenommen werden; darüber hinaus kann die Reklamation auch zu einem anderen Zeitpunkt von den Gesprächsbeteiligten wieder aufgenommen und weiter bearbeitet werden. Auch können Handlungsschemata mehrmals - meist verkürzt - durchlaufen und dabei weitere Aufgaben bearbeitet oder bereits bearbeitete Aufgaben erweitert behandelt werden. Bei der Analyse eines Gesprächs muss für jede Äußerung überprüft werden, welche Aufgabe mit ihr bearbeitet wird unter Berücksichtigung der Polyfunktionalität der Äußerungen. Mit dem Handlungsschema-Konzept können komplexe (Reklamations-)Gespräche sinnvoll phasiert werden. Probleme bei der Durchführung der interaktiven Aufgaben können deutlich gemacht werden und entsprechend Kommunikationskonflikte zwischen den Gesprächsbeteiligten als Folge einer fehlenden oder ungenügenden Bearbeitung von Aufgaben festgestellt und systematisiert werden, wie es Fiehler/Kindt/Schnieders (1999) aufgezeigt haben.

Für eine ganze Reihe weiterer Gesprächstypen wurden Handlungsschemaanalysen durchgeführt und herausgearbeitet, so für Beratungsgespräche (Kallmeyer 1985; Nothdurft 1984; Nothdurft/Reitemeyer/Schröder 1994), Schlichtungsgespräche (Nothdurft 1995; Nothdurft/Spranz-Fogasy 1991), Ver- kaufsgespräche (Pothmann 1997), familiale Konfliktgespräche, Streit (Spranz-Fogasy/ Fleischmann 1993; Spiegel 1995), Arzt-Patienten-Interaktion (Nothdurft 1986b; SpranzFogasy 1988), Verhandlungen (Kallmeyer 1979), Therapiegespräche (Stitz/Speck/Gessinger 1987).

\section{Forschungsprobleme und Desiderate}

Der vorliegende Überblick lässt erkennen, dass typenspezifische, lineare Gesprächssegmentierungen mit Hilfe von thematischen und/oder handlungsschematischen Orientierungen empirisch problematisch sind, da die Modelle einer linearen und schrittweise prozessualen Abfolge von Gesprächsphasen nicht der Gesprächsrealität entsprechen und die Organisationsanstrengungen von $\mathrm{Ge}-$ sprächsteilnehmern unzulässig verkürzen.

Insbesondere gibt es für die Gesprächsmitte, also für die Kernphase von Gesprächen, im Vergleich zu den stark routinisierten und z. T. auch ritualisierten Gesprächsphasen der Eröffnung und Beendigung noch wenig befriedigende Phasierungsmodelle. Eine Ausnahme bilden manifest aufgabenstrukturierte Interaktionen, die entlang des Handlungsschema-Modells beschrieben werden können. Ein vergleichbares Modell für themenbezogen emergent strukturierte Interaktionstypen wie Diskussionen oder small talk liegt dagegen derzeit noch nicht vor.

Andere Ebenen der Interaktion, die neben den themen- und handlungsbezogenen Phasierungen ebenfalls phasische Strukturierungen erzeugen, wie beispielsweise die Ebene der Interaktionsmodalität oder die Identitätsund Beziehungsebene, blieben für Phasierungsmodelle bislang gänzlich unberücksichtigt. So sind bspw. in konfliktären Gesprächen wie Streits mit sich abwechselnden Eskalations- und Deeskalationsphasen Aspekte der Gesprächsmodalität und der Beziehungskonstitution oft aufschlussreicher für die Rekonstruktion phasischer Strukturierung als die damit einhergehenden thematischen oder handlungsorganisatorischen Entwicklungen (s. Spiegel 1995). Daraus abzuleiten ist dann als Forschungsdesiderat, neben der Untersuchung einzelner Ebenen, auch die Verflechtung der verschiedenen Ebenen und ihre Interdependenz bei der Konstitution einer Phasenstruktur deutlicher herauszustellen. 


\section{Literatur (in Auswahl)}

Atkinson, J. Maxwell/Heritage, John (1984): Topic organization. In: Atkinson, J. Maxwell/Heritage, John (eds.): Structure of social action. Cambridge, $165-166$

Beaugrande, Robert de/Dressler, Wolfgang (1981): Einführung in die Textlinguistik. Tübingen.

Becker-Mrotzek, Michael (1999): Diskursforschung und Kommunikation. 2. Aufl. Heidelberg.

Berens, Franz J. (1981): Dialogeröffnung in Telefongesprächen: Handlungen und Handlungsschemata der Herstellung sozialer und kommunikativer Beziehungen. In: Schröder, Peter/Steger, Hugo (eds.): Dialogforschung. Jahrbuch 1980 des Instituts für deutsche Sprache. Düsseldorf, 402-417.

Bergmann, Jörg R. (1990): On the local sensitivity of communication. In: Markova, Ivana/Foppa, Klaus (eds.): The dynamics of dialogue. New York, 201-226.

Brinker, Klaus (1988): Thematische Muster und ihre Realisierung in Talkshowgesprächen. In: Zeitschrift für Germanistische Linguistik 16, 26-45.

Brinker, Klaus/Sager, Sven F. (1996): Linguistische Gesprächsanalyse - Eine Einführung. 2. Aufl. Berlin.

Brons-Albert, Ruth (1995): Auswirkungen von Kommunikationstrainings auf das Gesprächsverhalten. Tübingen.

Brünner, Gisela/Fiehler, Reinhard/Kindt, Walther (eds.) (1999): Angewandte Diskursforschung. Band 1. Grundlagen und Beispielanalysen. Opladen/ Wiesbaden, $120-154$.

Deppermann, Arnulf (1999): Gespräche analysieren. Eine Einführung in konversationsanalytische Methoden. Opladen.

Dijk, Teun A. van (1980a): Textwissenschaft. Eine interdisziplinäre Einführung. Tübingen.

- (1980b): Macrostructures. An interdisciplinary study of global structures in discourse, interaction and cognition. Hillsdale, N. J

- (1982): Episodes as units of discourse analysis. In: Tannen, Deborah (ed.): Analyzing discourse: Text and talk. Georgetown, 177-195.

Dittmar, Norbert (1988): Zur Interaktion von Themenkonstitution und Gesprächsorganisation am Beispiel des therapeutischen Diskurses. In: Linguistische Berichte 113, 64-85.

Ehlich, Konrad/Rehbein, Jochen (1972): Zur Konstitution pragmatischer Einheiten in einer Institution: Das Speiserestaurant. In: Wunderlich, Dieter (ed.): Linguistische Pragmatik. Frankfurt/M., 209-254

Ehlich, Konrad/Rehbein, Jochen (1979): Sprachliche Handlungsmuster. In: Soeffner, Hans G. (ed.): Interpretative Verfahren der Sozial- und Textwissenschaften. Stuttgart, 243-274.
Ehlich, Konrad/Rehbein, Jochen (1986): Muster und Institution: Untersuchungen zur schulischen Kommunikation. Tübingen.

Fiehler, Reinhard/Kindt, Walther/Schnieders, Guido (1999): Kommunikationsprobleme in Reklamationsgesprächen. In: Brünner, Gisela/Fiehler, Reinhard/Kindt, Walther (eds.): Angewandte Diskursforschung. Band 1. Grundlagen und Beispielanalysen. Opladen/Wiesbaden, 120-154.

Franke, Wilhelm (1985): Das Verkaufs-/Einkaufsgespräch: Entwicklung eines dialoggrammatischen Beschreibungskonzeptes. In: Wirkendes Wort 35, $53-72$.

Goffman, Erving (1971): Verhalten in sozialen Situationen. Bielefeld.

- (1977): Rahmen-Analyse. Ein Versuch über die Organisation von Alltagserfahrungen. Frankfurt/ M.

Hausendorf, Heiko (1992): Gespräch als System. Linguistische Aspekte einer Soziologie der Interaktion. Opladen.

Henne, Helmut/Rehbock, Helmut (1995): Einführung in die Gesprächsanalyse. 3. Aufl. Berlin.

Heritage, John (1985): Recent developments in conversational analysis. In: Sociolinguistics 15, $1-19$.

Hinnenkamp, Volker/Selting, Margret (eds.) (1989): Stil und Stilisierung. Arbeiten zur interpretativen Soziolinguistik. Tübingen.

Hundsnurscher, Franz/Franke, Wilhelm (eds.) (1985): Das Verkaufs-/Einkaufsgespräch: Eine linguistische Analyse. Stuttgart.

Jäger, Karl-H. (1976): Zur Beendigung von Dialogen. Überlegungen, Vorschläge und erste Systematisierungsversuche. In: Berens, Franz J./Jäger, Karl-H./Schank, Gerd u. a. (eds.): Projekt Dialogstrukturen. Ein Arbeitsbericht. München, 105135.

Kallmeyer, Werner (1978): Fokuswechsel und Fokussierungen als Aktivitäten der Gesprächskonstitution. In: Meyer-Hermann, Reinhard (ed.): Sprechen-Handeln-Interaktion. Ergebnisse aus Bielefelder Forschungsprojekten. Tübingen, 179250.

- (1979): Kritische Momente. Zur Konversationsanalyse von Interaktionsstörungen. In: Frier, Wolfgang/Labroisse, Gerd (eds.): Grundlagen der Textwissenschaft. Linguistische und literaturwissenschaftliche Aspekte. Amsterdam, 59-109.

- (1985): Handlungskonstitution im Gespräch. Dupont und sein Experte führen ein Beratungsgespräch. In: Gülich, Elisabeth/Kotschi, Thomas (eds.): Grammatik, Konversation, Interaktion. Tübingen, 81-122.

Kallmeyer, Werner/Schütze, Fritz (1976): Konversationsanalyse. In: Studium Linguistik 1, 1-28.

Kallmeyer, Werner/Schütze, Fritz (1977): Zur Konstitution von Kommunikationsschemata der Sach- 
verhaltsdarstellung. Exemplifiziert am Beispiel von Erzählungen und Beschreibungen. In: Wegner, Dirk (ed.): Gesprächsanalysen. Referate der IPKTagung 1976. Hamburg, 159-274.

Labov, William/Waletzky, Joshua (1973): Erzählanalyse: mündliche Versionen persönlicher Erfahrung. In: Ihwe, Jens (ed.): Literaturwissenschaft und Linguistik. Bd. 1. Frankfurt/M., 78-126.

Levinson, Stephen C. (1990): Pragmatik. Tübingen. Linke, Angelika/Nussbaumer, Markus/Portmann, Paul R. (1996): Studienbuch Linguistik. Tübingen.

Marten, S. (1985): Praxisbericht zum persönlichen Verkauf. In: Hundsnurscher, Franz/Franke, Wilhelm (eds.): Das Verkaufs-/Einkaufsgespräch: Eine linguistische Analyse. Stuttgart, 10-40.

Müller, Klaus (1984): Rahmenanalyse des Dialogs. Tübingen.

Nofsinger, Robert E. (1991): Everyday conversation. Newbury Park/London/New Delhi.

Nothdurft, Werner (1984): „... äh folgendes Problem äh ...": Die interaktive Ausarbeitung „des Problems" in Beratungsgesprächen. Tübingen.

- (1986a): Das Muster im Kopf? Zur Rolle von Wissen und Denken bei der Konstitution interaktiver Muster. In: Kallmeyer, Werner (ed.): Kommunikationstypologie: Handlungsmuster, Textsorten, Situationstypen. Düsseldorf, 92-116.

- (1986 b): Schilderung von Beschwerden in ärztlichen Sprechstundengesprächen. In: Löning, Petra/Sager, Sven F. (eds.): Kommunikationssystem ärztlicher Gespräche. Hamburg, 17-39.

- (ed.) (1995): Schlichtungsgespräche. Bd. 1. Streit schlichten. Gesprächsanalytische Untersuchungen zu institutionellen Formen konsensueller Konfliktregelung. Berlin/New York.

Nothdurft, Werner/Reitemeier, Ulrich/Schröder, Peter (1994): Beratungsgespräche. Analyse asymmetrischer Dialoge. Tübingen.

Nothdurft, Werner/Spranz-Fogasy, Thomas (1991): Gesprächsanalyse von Schlichtungs-Interaktion. Methodische Probleme und ihre Hintergründe. In: Flader, Dieter (ed.): Verbale Interaktion. Stuttgart, 222-240.

Pothmann, Achim (1997): Diskursanalyse von Verkaufsgesprächen. Opladen.

Putnam, Hilary (1958): Formalization of the concept „about“. In: Philosophy of Science 25, 125130.

Quasthoff, Uta (1980): Erzählen in Gesprächen. Tübingen.

Rehbein, Jochen (1977): Komplexes Handeln. Elemente zur Handlungstheorie der Sprache. Stuttgart.

- (1987): Ausgewählte Aspekte der Pragmatik. In: Ammon, Ulrich/Dittmar, Norbert/Mattheier, Klaus
J. (eds.): Sociolinguistics/Soziolinguistik. Second Vol./Zweiter Halbbd. Berlin/New York, 1181-1195.

- (1995): International sales talk. In: Ehlich, Kon$\mathrm{rad} /$ Wagner, Johannes (eds.): The discourse of business negotiation. Berlin, 67-102.

Sacks, Harvey (1971): Das Erzählen von Geschichten innerhalb von Unterhaltungen. In: Kjolseth, Rolf/Sack, Fritz (eds.): Zur Soziologie der Sprache. Kölner Zeitschrift für Soziologie und Sozialpsychologie. Sonderheft 5/1971, 307-314.

- (1992): Lectures on conversation. Oxford.

Schank, Gerd (1981): Untersuchungen zum Ablauf natürlicher Dialoge. München.

Schank, Roger C./Abelson, Robert P. (1977): Scripts, plans, goals and understanding. An inquiry into human knowledge structure. Hillsdale, N. J.

Schegloff, Emanuel A. (1968): Sequencing in conversational openings. In: American Anthropologist 70, 1075-1095.

Schegloff, Emanuel A./Sacks, Harvey (1973): Opening up closings. In: Semiotica 8, 289-327.

Schütze, Fritz (1978): Strategische Interaktion im Verwaltungsgericht - eine soziolinguistische Analyse zum Kommunikationsverlauf im Verfahren zur Anerkennung als Wehrdienstverweigerer. In: Interaktion vor Gericht. Mit Beiträgen von HoffmannRiem, Wolfgang/Rottleuthner, Hubert/Schütze, Fritz/Zielcke, Andreas. Baden-Baden, 19-100.

Spiegel, Carmen (1995): Streit. Eine linguistische Untersuchung verbaler Interaktionen in alltäglichen Zusammenhängen. Tübingen.

Spranz-Fogasy, Thomas (1988): Medikamente im Gespräch zwischen Arzt und Patient. In: Deutsche Sprache 3, 240-258.

- (1997): Interaktionsprofile. Die Herausbildung individueller Handlungstypik in Gesprächen. Opladen.

Spranz-Fogasy, Thomas/Fleischmann, Thomas (1993): Types of Dispute Courses in Family Interaction. In: Argumentation 7, 221-235.

Stitz, Sabine/Speck, Agnes/Gessinger, Joachim (eds.) (1987): Therapiegespräche. Bremen.

Werlen, Iwar (1984): Ritual und Sprache. Zum Verhältnis von Sprechen und Handeln in Ritualen. Tübingen.

Techtmeier, Bärbel (1984): Das Gespräch. Funktionen, Normen und Strukturen. Berlin.

Wolf, Ricarda (1991): „Stopp ma bitte, wir müssen mal weiter zum Kern kommen!" Zur Rolle von Metakommunikation bei der Thema-Entwicklung - untersucht an Problemlösungsgesprächen einer Musiker-Gruppe. Diss. Potsdam.

Carmen Spiegel, Heidelberg (Deutschland) Thomas Spranz-Fogasy, Mannheim

(Deutschland) 